\title{
Real-time Sampling Rate Adaptation based on Continuous Risk Level Evaluation in Wireless Body Sensor Networks
}

\author{
Carol Habib ${ }^{a, b}$, Abdallah Makhoul ${ }^{a}$, Rony Darazi ${ }^{b}$, Raphaël Couturier $^{a}$ \\ ${ }^{a}$ Femto-st institute, Univ. Bourgogne Franche-Comté, Belfort, France \\ ${ }^{b}$ TICKET Lab, Antonine University, Hadat-Baabda, Lebanon \\ Emails: carol.habib@univ-fcomte.fr, abdallah.makhoul@univ-fcomte.fr, \\ rony.darazi@ua.edu.lb,raphael.couturier@univ-fcomte.fr
}

\begin{abstract}
Wireless Body Sensor Networks (WBSNs) are a lowcost solution allowing remote patient monitoring and continuous health assessment, thus reducing healthcare expenditure. In such networks, sensor nodes periodically collect vital signs and send them to the coordinator for fusion. However, sensor nodes have limited energy and processing resources and transmission is the most power-hungry task. In this paper, we target data reduction and energy consumption. We propose to locally adapt, in real-time, the sampling rate of a sensor node according to the variations in the vital sign being monitored and its risk. We propose to dynamically evaluate, in real-time, the risk of any vital sign given the information about the severity level of the patient's health condition and the severity level of the vital sign itself. We have tested our proposed approach on real health datasets in order to evaluate it. The results show that the percentage of detected critical events and the mean-square error (MSE) are both acceptable. In addition, the percentage of data reduction is around $50 \%$ implying a reduction of the energy consumption. Adjusting the risk of a vital sign, over time, ensures the adaptation of the sampling rate according to the overall health condition of the patient as well as the severity level of the collected measurements.
\end{abstract}

Index Terms-Adaptive sampling, risk level evaluation, behavior function, health assessment, wireless body sensor networks

\section{INTRODUCTION}

In the past decade, Wireless Body Sensor Networks (WBSNs) emerged as a low cost solution for remote and continuous patient monitoring, thus reducing unnecessary hospitalization as well as keeping doctors and the healthcare center up-to-date with health-related matters [1]. Many populations can benefit from such a technology: elderly in nursing homes, patients at home after a surgical intervention, patients susceptible to cancer or heart disease, athletes etc. Some of the most popular applications in the literature include fall detection, stress evaluation systems, gait analysis, monitoring of vital signs and physilogical signals [2], [3], [4]. A WBSN is composed of sensor nodes and a coordinator. The former are placed on the body of the patient. They sense physiological signals, extract vital signs and wirelessly send these measurements to the coordinator for fusion in a periodic fashion. We suppose that each sensor node monitors only one vital sign (e.g. temperature, respiration rate, etc.). The coordinator usually is the smartphone, PDA or any other portable device which is carried by the patient. Many challenges exist in such a network such as: the heterogeneity of the collected data (heart rate, respiration rate, blood pressure etc.) and its huge amount, the energy consumption due to periodic transmission as well as privacy and security issues [1], [5], [7]. In this paper we address the energy consumption and data reduction issues which are directly related to one another. In [6], the authors have classified energy-efficient mechanisms in Wireless Sensor Networks (WSNs) into five categories: data reduction approaches, sleep/wakeup schemes, radio optimization techniques, energyefficient routing methods and battery repletion. These approaches can be split up into software and hardware strategies [8]. In addition, some of these mechanisms are suitable for large scale networks such as environmental monitoring, industry, public safety or military systems applications. Thus, they cannot be applied in WBSNs where the network characteristics are different. For example, energy-efficient routing methods as well as transmission power control and topology control approaches cannot be directly used in WBSNs [9]. Many existing approaches in WBSNs have proposed energy-efficient data collection approaches. Some have used context-awareness based on activity recognition to perform adaptive sampling or adaptive sensing [8]. Some of them applied these approaches only on WBSNs composed of inertial detection sensor nodes such as accelerometers and gyroscopes [9]. Others suggested adapting the transmission of sensor nodes according to the relevance between symptoms and diseases [10]. Others have used compressive sensing (CS) theory, which has emerged as an energy-efficient approach for wireless communication, due to the fact that biosignals such as the electrocardiogram (ECG) are sparse [11], [12], [13], [14]. This technique reduces the amount of wirelessly transmitted data by sampling the signal of interest at a much lower rate than the traditional ShannonNyquist theorem. Thus, it has the potential of dramatically reducing the power consumption due to transmission. However, this technique has not been applied at the analog level since it requires extensive work on the analog sensor read-out electronics prior to the analog-to-digital converter (ADC). Its application on the digital level needs processing and energy 
resources and depends on the sensor's microcontroller [11]. In addition, it requires the sparsity of the signals of interest, thus in case of non-sparstity it does not perform as desired [12]. In [15], the authors have proposed AdapDBN a selfadaptively sepsis screening system allowing to shorten the time of syndrome detection. In a previous work ([16]), a distributed self-adaptive data collection approach has been proposed in the context of WBSNs. An adaptive sampling rate scheme, having a direct impact on the sensing, processing and especially the transmission tasks of the biosensor node, has been proposed. Using a Quadratic Bezier Curve as a BehaVior (BV) Function, it takes into account two parameters : the evolution of the monitored vital sign over time and its monitoring importance, based on a medical judgment, regarding the patient's health condition. These parameters are, respectively, determined by the Fisher Test and one-way Analysis of Variance (ANOVA) which study the variances of the sensed measurements over time and by a static risk level variable. However, the overall health condition of a patient, being continuously and remotely monitored on a long-term basis, changes over time. It is subject to many health events which can be acute or even chronic. Thus, it can vary from day to day as well as from an improvement state into a deterioration state and vice versa especially that acute disease go through many different stages. As a consequence, the monitoring importance given for each vital sign should be adapted with these changing conditions. This matter, has a direct influence on the collection of data, therefore on the energy consumption of the WBSN and the early detection of critical events. In this paper, we propose to dynamically adapt the risk level of a vital sign according to the changing health condition of the patient. The remainder of the paper is organized as follows. Section II presents our work's background. The risk level of the sensor node is defined and its adaptation is explained in Section III. Experimental results are shown and discussed in Section IV. Finally Section V concludes the paper with some directions and future work.

\section{BACKGROUND}

In this section, a brief overview about early warning score systems is given and the BehaVior (BV) function is extensively explained by adapting its equations based on the requirements of vital signs monitoring. The former is used to evaluate the patient's overall health condition as well as the severity of each vital sign, whereas, the latter is used for the sampling rate adaptation.

\section{A. Early Warning Score Systems}

An early warning score system (EWS) is a chart used by emergency medical services staff in hospitals to evaluate patients' heath status. The vital signs are weighed and aggregated in order to allow an early recognition of patients who are subject to an acute illness or those whose health condition is deteriorating [17]. For each vital sign, a normal healthy range is defined. Values outside of this range are allocated a score according to the magnitude of the deviation from the normal range. The score weighing reflects the severity of the physiological disturbance. Such scoring systems can give the biosensor node the ability to locally evaluate the severity level of the vital sign being monitored and to assign to it a score. We have used the National EWS (NEWS) in our work in order to evaluate the severity level of each of the following vital signs: temperature, heart rate, respiration rate, oxygen saturation and systolic blood pressure. A score of 0 is assigned to the measurement if it is in the normal range and a score of 1,2 or 3 , according to its magnitude of deviation from the normal range, where 3 indicates the highest level of criticality. NEWS is standarized and employed in hospitals in the United Kingdom (UK) for the assessment of accute-illeness [18].

\section{B. Behavior Function}

Bezier curves are flexible parametric curves allowing the definition of shapes given the knowledge about some points of interest [19]. The quadratic bezier curves, which curvature is defined following three points of interest, are used in the proposed sampling adaptation model. A quadratic bezier curve is limited by the two points $P_{0}\left(l_{x} ; l_{y}\right)$ and $P_{2}\left(h_{x} ; h_{y}\right)$ and its curvature is controlled by $P_{1}\left(b_{x} ; b_{y}\right)$. It can be written under the following general form:

$$
a x^{2}+b x y+c y^{2}+u x+v y+w=0
$$

where the values of the coefficients depend on $P_{0}, P_{1}$ and $P_{2}$.

In our approach, the $\mathrm{x}$-axis represents the Fisher Test result $F$ and the y-axis represents the sampling rate $S R$. Thus, the three points of the $\mathrm{BV}$ function are defined as follows: $P_{0}\left(0 ; l_{y}\right), P_{1}\left(b_{x} ; b_{y}\right), P_{2}\left(h_{x} ; h_{y}\right)$ where $P_{0}$ corresponds to $F=0$ and $S R=S R_{\min }, P_{1}$ is the behavior point and $P_{2}$ corresponds to $F=F_{t}$ and $S R=S R_{\max }$. $F_{t}(h, N-h)$ corresponds to the critical value given by the Fisher Test for $N$ collected samples during $h$ periods. The BV function curve can be drawn using the quadratic bezier curve as follows:

$$
B V(F)= \begin{cases}\frac{h_{x}+l_{y}-2 b_{y}}{4 b_{x}^{2}} F^{2}+\frac{h_{y}-l_{y}}{h_{x}} F+l_{y}, & h_{x}=2 b_{x} \\ \left(h_{y}+l_{y}-2 b_{y}\right) \alpha(F)^{2}+ & \\ 2\left(b_{y}-l_{y}\right) \alpha(F)+l_{y}, & \text { otherwise }\end{cases}
$$

with $\alpha(F)=-b_{x}+\frac{\sqrt{b_{x}^{2}-2 b_{x} F+h_{x} F}}{h x-2 b x}$, such as $0 \leq b_{x} \leq h_{x}$, $0 \leq F \leq h_{x}$ and $h_{x}>0$.

$P_{1}$ moves on the diagonal $[\mathrm{AB}]$ of the behavior rectangle, such as $A\left(0 ; h_{y}\right)$ and $B\left(h_{x} ; l_{y}\right)$. Thus, the coordinates $b_{x}$ and $b_{y}$ of $P_{1}$ satisfy the equation of $[\mathrm{AB}]$ which is defined as follows:

$$
y=\frac{l_{y}-h_{y}}{h_{x}} \times x+h_{y}
$$

In our approach, the position of $P_{1}$ should be determined according to the value of the risk level $r$. The coordinates $b_{x}$ and $b_{y}$ of $P_{1}$ are found using the following equation: 


$$
\begin{aligned}
B_{r}:[0 ; 1] & \longmapsto\left[0 ; h_{x}\right] \times\left[l_{y} ; h_{y}\right] \\
r & \longmapsto\left(b_{x} ; b_{y}\right)
\end{aligned}
$$

Thus, the following equations can be derived:

$$
B_{r}(r)=\left\{\begin{array}{l}
b_{x}=(1-r) \times h_{x} \\
b_{y}=l_{y}+r \times\left(h_{y}-l_{y}\right)
\end{array}\right.
$$

The closer the value of $r$ is to 1 the more the vital sign is judged to be critical and its monitoring importance is increased. Figure 1 shows the BV function which is used to adapt the sampling rate according to the Fisher test result $F$ and the risk level $r$ of the vital sign given the patient's health condition. Having a maximum sampling rate $S R_{\max }$ and a minimum sampling rate $S R_{\text {min }}$, depending on the application's requirements, and the critical value $F_{t}$ given by the Fisher Test, the BV function is defined as follows:

$$
B V\left(S R_{\text {max }}, S R_{\text {min }}, r, F, F_{t}\right)=S R
$$

The closer $F$ is to $F_{t}$, the more the collected measurements in $h$ periods present variations. Thus, the higher the sampling rate is given to the sensor node in order not to miss any important variations. Whereas, the closer $\mathrm{F}$ is to 0 , the less the collected measurements in $h$ periods present variations. Thus, a lower sampling rate is given to the sensor node in order to preserve their energy level. A curve is associated for each risk level value. The higher the risk level value, the greater the sampling rate values. Indeed, for high risk levels the sampling rate of the sensor node should be increased in order not to miss any important changes and to keep track with the variations of the corresponding vital sign.

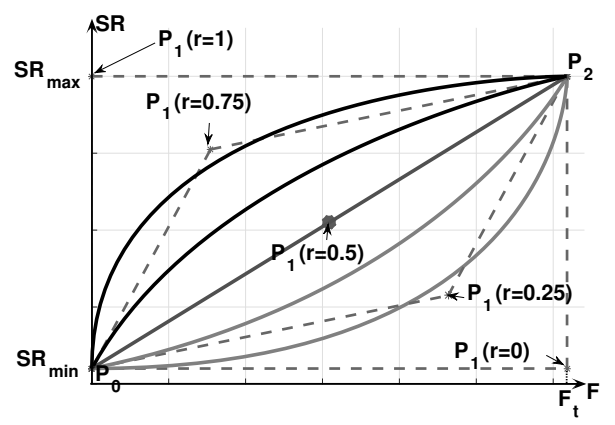

Fig. 1: Behavior Function: Quadratic Bezier Curves

As shown in Figure 2, for the same value of $F$, if the vital sign has a low risk level $r$, the sensor's measurement sampling rate will take a lower value than if the vital sign has a high risk level $r$. Thus, in the first case, the biosensor node will preserve its energy and will sense data and process measurements at a lower rate. However, in the latter case, the biosensor node, which is monitoring a vital sign being medically judged as essential regarding the patient's health condition, will be assigned a higher sampling rate in order not to miss important measurements and events.

In the following section, we describe the risk level adaptation of a given vital sign according to the dynamic change of

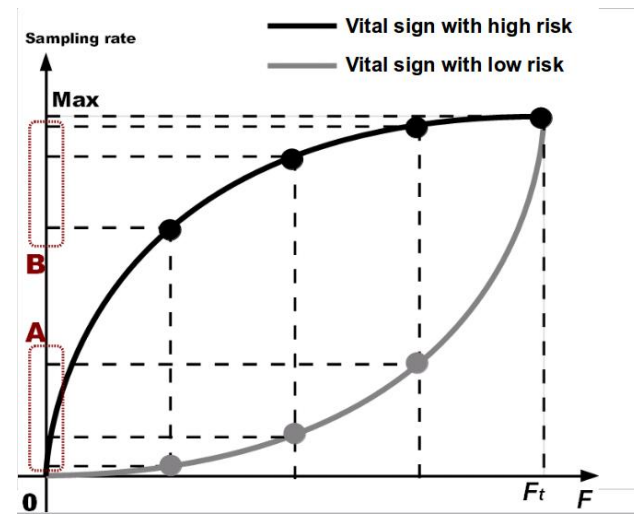

Fig. 2: Sampling rate adaptation according to the vital sign's risk level

the patient's health condition over time. The sensor node will be able to adapt its sampling rate based on the BV curve that corresponds to its dynamically adapted risk level.

\section{Risk LEVEL ADAPTATION}

\section{A. Scenario}

Tom is an elderly person living in a nursing home. In order to keep track of his health condition, he is being remotely and continuously monitored by a WBSN. We suppose that the WBSN is composed of $n$ biosensor nodes, where each node monitors one vital sign. At first, the monitoring importance for each vital sign is medically judged by the medical team. Accordingly, the risk levels $r$ for all the vital sign are chosen. Since Tom's health condition can change with time, he can become sick or be subject to dangerous health events. Thus, the risk levels $r$ should be adapted by the WBSN with Tom's changing health condition. Higher values should be given to the nodes in order to increase their sampling rate and capture important measurements and when the patient's health condition is at a lower risk and when it is normal, lower risk values should be given to the nodes in order to preserve their energy level. Next, we give a proper definition to the risk level $r$ and discuss its initial setup.

\section{B. Definition of the risk level $r$}

The variable $r$ represents the monitoring importance given to a specific vital sign. At first, healthcare experts chose the values for all the monitored vital signs. $r$ is assigned a low value $(<0.5)$ if the following conditions are met:

- If the vital sign generally does not present many variations and is usually stable.

- If the medical expert thinks that a low sampling rate is sufficient to capture any important variation.

- If the variations do not have a drastic impact on the patient's health.

For example, the temperature, the galvanic skin response and the oxygen saturation meet these requirements.

$r$ is assigned a high value $(\geq 0.5)$ if the following conditions are met: 
- If the vital sign usually presents many variations.

- If the medical expert thinks that a high sampling rate is essential to capture any important variation.

- If any variation can have a drastic impact on the patient's health.

For example, the heart rate, the blood pressure and the respiration rate meet these requirements.

\section{Risk Level Evaluation Function}

We propose to adapt the risk level $r$ of a vital sign, throughout the life of the WBSN, according to the patient's changing health condition. Let $r_{\text {global }}$ represent the patient's overall health condition where $r_{\text {global }} \in[0 ; 1]$ and $r_{\text {global }} \in \mathbb{R}$. $r_{\text {global }}$ is referred as the patient's global risk level and it indicates the severity level of his/her health condition. $r_{\text {global }}$ is evaluated by the coordinator based on the multi-sensor data fusion [20]. Let $\bar{S}$ be the average score of the sensed measurements for a given vital sign during one round $R$, such as $R=h \times p$ where $p$ is a time period and $h \in \mathbb{N}$, and $S_{\max }$ be the maximum score that a measurement can have according to the used EWS (cf. Section II-A). Then, the risk level evaluation function Eval $:\left(r_{\text {global }}, \bar{S}\right) \longmapsto[0 ; 1]$ is defined as follows:

$$
\operatorname{Eval}\left(r_{\text {global }}, \bar{S}\right)=\alpha \times r_{\text {global }}+\beta \times \frac{\bar{S}}{S_{\max }}
$$

where $\alpha$ and $\beta$ are weight coefficients such as : $0 \leq \alpha \leq 1$ and $0 \leq \beta \leq 1$ and $\alpha+\beta=1$. The Score ratio $\frac{\bar{S}}{S_{\max }}$ is equal to the mean score of the sensed measurements over the maximum score that can be given to a measurement. It represents the situation of a vital sign (its criticality) compared to the worst case (highest criticality level). $\bar{S}$ is calculated as follows:

$$
\bar{S}=\frac{1}{m} \times \sum_{i=1}^{m} s_{i}
$$

where $m$ is the total number of sensed measurements and $s_{i}$ is the score of th $i^{\text {th }}$ measurement.

Figure 3 shows the output of the proposed risk level evaluation function for two different parameter setups. The blue surface corresponds to a parameterization of $\alpha=\beta=0.5$, whereas the pink surface corresponds to a parameterization of $\alpha=0.2$ and $\beta=0.8$. In the former case, both the score ratio and the overall health condition of the patient are given equal weights. Thus, both are equally important. In the latter case, the score ratio is given a greater importance than the overall health condition. For example, for both cases, the lower the global risk and the higher the score ratio, then the higher the risk level. However, in the latter case the risk level will have higher values than the former case for the same global risk value, since the impact of the score ratio is greater than the impact of the global risk. The $\alpha$ and $\beta$ values are to be judged and parametrized by the healthcare experts, depending on whether the overall health condition of the patient or the status of the vital sign itself is more essential given the monitoring needs. For instance, some viruses such as flu or infections are accompanied by fever. Therefore, the temperature should be given a higher monitoring importance given its impact on the these types of sickeness and given that healthcare experts are interested in monitoring its variations over time. Thus, $\beta$ better have a higher value than $\alpha$ in order to give a higher importance to the score ratio of the temperature rather than the overall health condition regrouping all the vital signs being monitored.

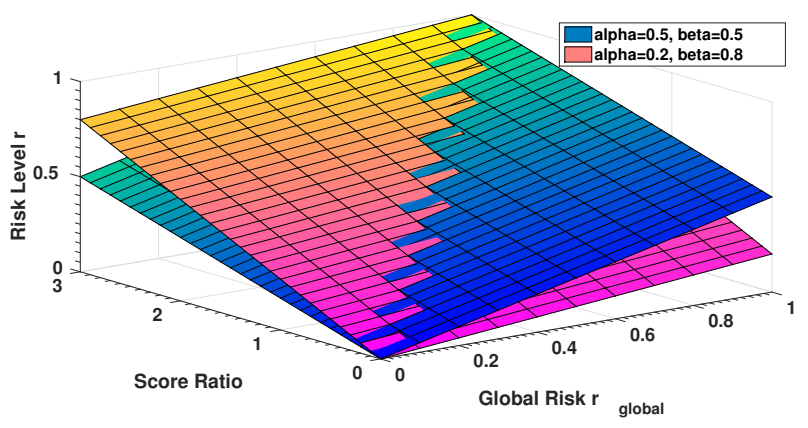

Fig. 3: Risk Level Evaluation Function

\section{Adaptive sampling using risk level evaluation algorithm}

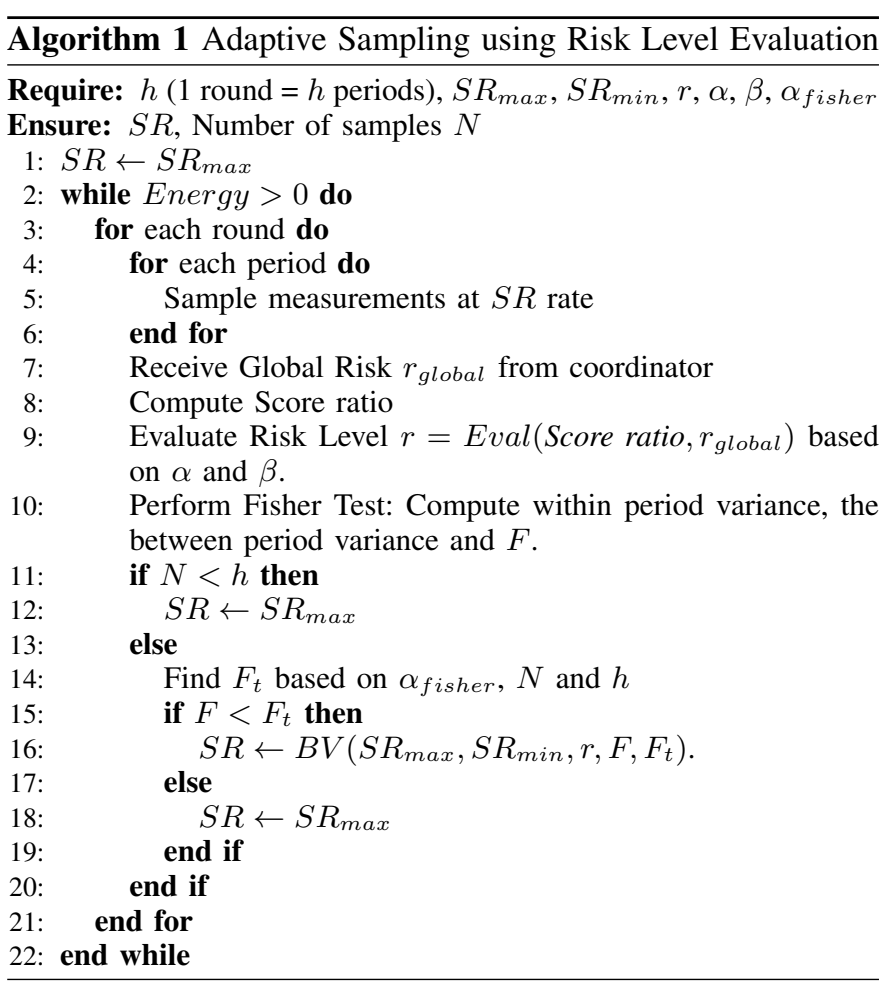

In this section, the algorithm for adapting the sampling rate in real-time is presented. The proposed algorithm is to be locally performed on each sensor node of the WBSN. The network is managed by one coordinator. Each sensor node locally adapts its sampling rate after adapting its risk level $r$ using the proposed risk level evaluation function. On one side, for each round, the coordinator sends the value of $r_{\text {global }}$, which is evaluated based on multisensor data fusion, to all the sensor nodes. On the other side, for each round, each sensor 
nodes computes the score ratio and evaluate its risk level based on these two values. Then, the sampling rate adaption can be made based on the result of Fisher test $F$ and $r$ using the $\mathrm{BV}$ function. The sampling rate adaptation is performed each round $k$ such as Round $_{k}=\sum_{i=k}^{h+k-1} p_{i}$ where $k \in \mathbb{N}, h \in \mathbb{N}$ and $p$ is the time period. In other words, it is performed at end of each period based on the measurements collected during the last $h$ periods. The global risk $r_{\text {global }}$, which is sent by the coordinator to the sensor nodes, is based on the data fusion of the collected measurements during these last $h$ periods.

\section{EXPERIMENTAL RESULTS}

Experiments are conducted using a cutom-based Java simulator. In order to evaluate the performance of the proposed approach, patient vital signs datasets are collected from Multiple Intelligent Monitoring in Intensive Care (MIMIC) I and II databases of PhysioNet [21]. We have tested our approach on different patient records as well as different vital signs such as the heart rate (HR), the respiration rate (RESP), the systolic blood pressure (ABPsys), the blood temperature (BLOODT) and the oxygen saturation $(\mathrm{SpO} 2)$. We suppose that each vital sign is monitored by only one sensor node: if $N$ vital signs are required to be monitored, then $N$ sensors nodes are deployed on the patient's body. The proposed algorithm (cf. Algorithm 1 ) is implemented and tested at the level of each node. After running multiple experiments, noting that a minimum of 30 samples is required for the Fisher Test and ANOVA, the parameters settings are chosen as follows:

- Period $p=100 \mathrm{sec}$ and Round $R=2 \times p$.

- Minimum sampling rate $S R_{\min }=1$ samples $/ 3.33 \mathrm{sec}$ (corresponding to 30 samples per period) and Maximum sampling rate $S R_{\max }=1 \mathrm{sample} / 1.42 \mathrm{sec}$ (corresponding to 70 samples per period).

- Fisher Risk $\alpha_{\text {fisher }}=0.05$.

- Initial risk level on all sensor nodes is randomnly set to $r^{0}=0.4$, indicating that all vital signs are slightly critical and have the same impact on the patient's health.

The global risk level which represents the overall health condition of the patient is determined by the coordinator by running the algorithm Health-RAD [22] for the multi-sensor data fusion and health assessment [20].

First, we show the risk adaptation over time for different $\alpha$ and $\beta$ setups and discuss their impact on the risk evaluation. Then we compare the results obtained in terms of data reduction, loss of information and energy consumption for 3 different scenarios : static risk level $r=0.4$, static risk level $r=0.9$ and our proposed approach.

\section{A. Risk Adaptation over time}

Figure 4 shows the ABPSys sensor node's risk level adaptation over 70 periods for 3 different parameter setups. In our proposed approach, $\alpha$ and $\beta$ respectively denote the weights accorded for the patient's overall health condition and for the vital sign's severity level. Initially, the sensor node's risk level is set to 0.4 , it is clear to see that in the 3 cases the risk evaluation function has yielded most of the time a risk value different than 0.4 and whose values range from low to high risk levels. Hence, pointing out the importance of dynamically adapting the risk level of each sensor node according to the changes in the patient's health conditon. When comparing Figures $4 \mathrm{a}$ and $4 \mathrm{~b}$, we can clearly see the influence of both parameters on the risk level evaluation. For example, between period 45 and period 60 , the risk level is mainly between 0.4 and 0.6 for $\alpha=0.7$ and $\beta=0.3$, however it ranges between 0.6 and 0.8 for $\alpha=0.3$ and $\beta=0.7$. This is due to the fact that between these periods the severity level of the ABPSys represented by the score ratio is more critical than the patient's overall health condition. Hence, giving the score ratio a higher impact than the overall health condition has yeilded a higher risk level (see Figure 4c) than in the opposite case (see Figure $4 \mathrm{~b})$. Whereas, between periods 8 and 18, the results show that the overall health condition is more critical than the severity level of the ABPSys. The risk level ranges between 0.2 and 0.4 , in the case of $\alpha=0.7$ and $\beta=0.3$ (see Figure 4b), however it ranges between 0.1 and 0.2 in the case of $\alpha=0.3$ and $\beta=0.7$ (see Figure 4c). Hence, the parameters $\alpha$ and $\beta$ of the risk level evaluation function should be medically judged by the healthcare experts on the basis of the importance given to each of the vital sign's severity level and the patient's overall health condition. In the rest of this section, the results correspond to a setup of $\alpha=\beta=0.5$.

\section{B. Sampling Rate Adaptation and Energy Consumption}

In this section, we compare the sampling rate adaptation and the energy consumption between two cases: static (constant) risk level $r$ and our proposal of dynamic risk level $r$. We study the sampling rate adaptation and the energy consumption of the HR sensor node over 70 periods for 2 different patients. Patient 1 corresponds to record s01840-3454-10-24-18-46nm from MIMIC II database whereas patient 2 corresponds to record 276n from MIMIC database. According to the available datasets, all of the 5 vital signs are monitored for patient 1 and only the HR and the ABPSys are monitored for patient 2.

1) Sampling Rate Adaptation: Figure 5 shows the sampling rate adaptation of the HR sensor node for both patients for 3 different cases: static risk $\mathrm{r}=0.4$, static risk $\mathrm{r}=0.9$ and dynamic risk. For both patients, when comparing the two static cases (see Figures 5b, 5c, 5e, 5f), we can notice that the sampling rate adaptation results in higher sampling rates when $r=0.9$ than when $r=0.4$. For example periods 2 and 10 in Figures $5 \mathrm{~b}, 5 \mathrm{c}$ and from period 50 till period 70 in Figures $5 \mathrm{e}$ and $5 \mathrm{f}$. This is due to the definition of the BV function which results in higher sampling rates for higher risk level values. However, if we compare the results obtained in the dynamic risk level case with the results of the two static cases for patient 1, we can notice that the total of sampled measurements during the 70 periods are less than the total of sampled measurements in the case of the static risk $r=0.9$ and slightly less than the case of static risk $r=0.4$. This indicates that our proposed approach has evaluated the HR risk level to less than 0.9. Thus, the amount of sampled data is reduced during the 70 periods which will have an impact on the energy consumption. 


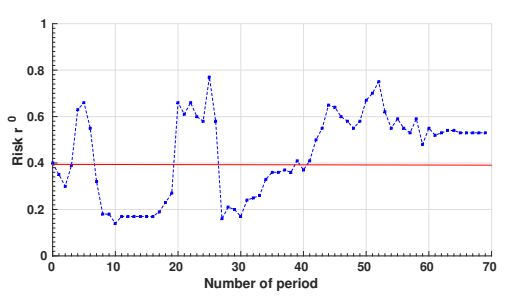

(a) $\alpha=\beta=0.5$

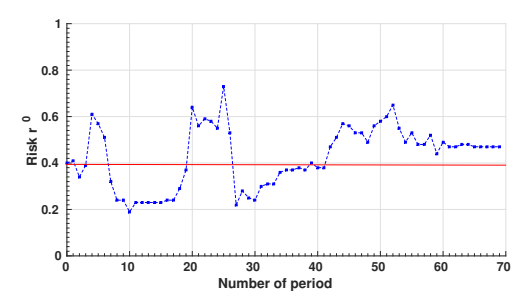

(b) $\alpha=0.7$ and $\beta=0.3$

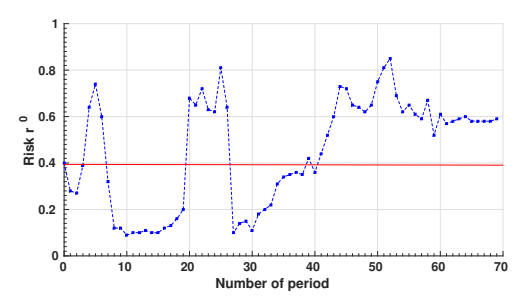

(c) $\alpha=0.3$ and $\beta=0.7$

Fig. 4: ABPSys sensor node's risk level adaptation over 70 periods with different $\alpha$ and $\beta$ setups

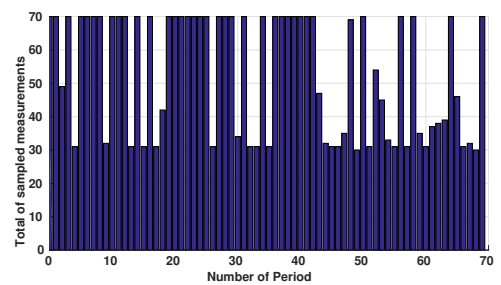

(a) Patient 1: Dynamic Risk

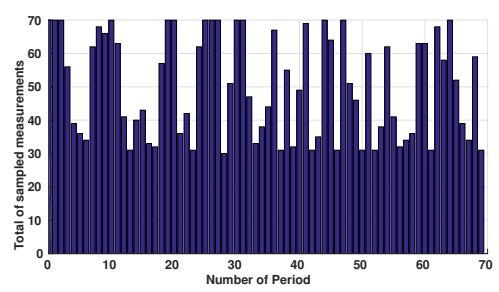

(d) Patient 2: Dynamic Risk

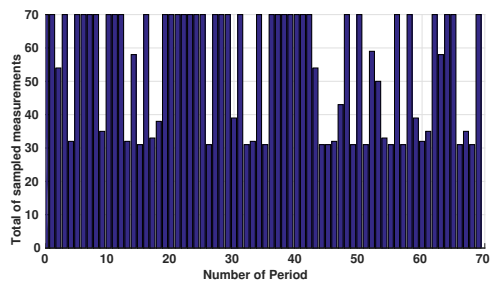

(b) Patient 1: Static Risk $r=0.4$

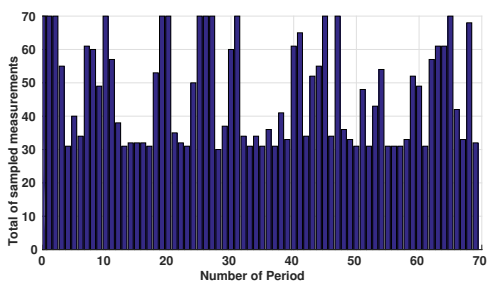

(e) Patient 2: Static Risk $r=0.4$

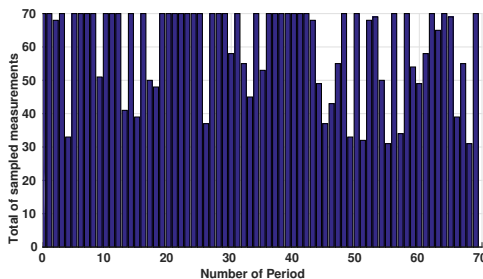

(c) Patient 1: Static Risk $r=0.9$

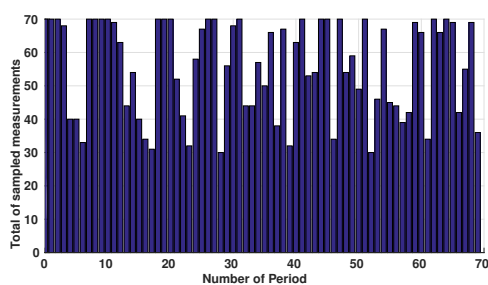

(f) Patient 2: Static Risk $r=0.9$

Fig. 5: Comparison of HR sensor node's sampling rate adaptation over 70 periods between 3 different cases for 2 different patients.

While, if we compare the results obtained for patient 2 , we can notice that the amount of sampled measurements in the case of dynamic risk is greater than the amount of samples in the case of static risk $r=0.4$ and less than the amount of samples in the case of static risk $r=0.9$. This indicates that our proposed approach has evaluated the HR risk level less than 0.9. Thus, the amount of sampled data is reduced during the 70 periods compared to a static risk of 0.9 and slighlty increased compared to a static risk of 0.4. Therefore, adapting the risk level value over time has an impact on the Fisher test result since the amount of sampled data changes from one period to another, as well as on the BV function and thus on the output of the sampling rate adaptation scheme. This will have an impact on the energy consumption.

2) Energy Consumption: Figure 6 shows the HR sensor node's remaining energy at the end of 70 periods for both patient 1 and 2. We suppose that 1 unit of energy is equal to 152 Joules : the sensing task consumes 6 Joules, the processing task consumes 24 Joules, the transmission task (TX) consumes 60 Joules and the receiving task (RX) consumes 62 Joules [23]. Thus, we suppose that each sampled measurement needs 0.6 units of energy to be sent to the coordinator. It includes 0.04 units of energy for the sensing task, 0.16 units of energy for the processing task and 0.4 units of energy for the transmission task. Having the initial energy randomnly set to 4000 units, the dynamic approach has consumed the least energy among the 3 cases in the case of patient 1 . The remaining energy is 1463.8 units when setting the risk level to 0.9 , is 1687 units when setting the risk level to 0.4 and 1753.6 units when adapting the risk level over the 70 periods. However, the results of the HR sensor node's sampling rate adaptation of patient 2 shows that adapting the risk level over time has reduced the energy consumption compared to setting the risk level value to 0.9 but it has increased the energy consumption compared to setting the risk level value to 0.4 . The remaining energy is 1703.2 units when setting the risk level to $0.9,2031.4$ units when setting the risk level to 0.4 and 1888.6 units when adapting the risk level over the 70 periods. Thus, adapting the risk level of a vital sign over time adapts the lifetime of a sensor node to the patient's changing health condition.

\section{Data Reduction vs Loss of information}

In this section, we study the data reduction performed at the sensor node level following the sampling rate adaptation and discuss its impact on the loss of information. We compare the results obtained for the adaptive sampling rate scheme with static risk level and with dynamic risk level for patient s01840-3454-10-24-18-46nm. Table I shows the data reduction 


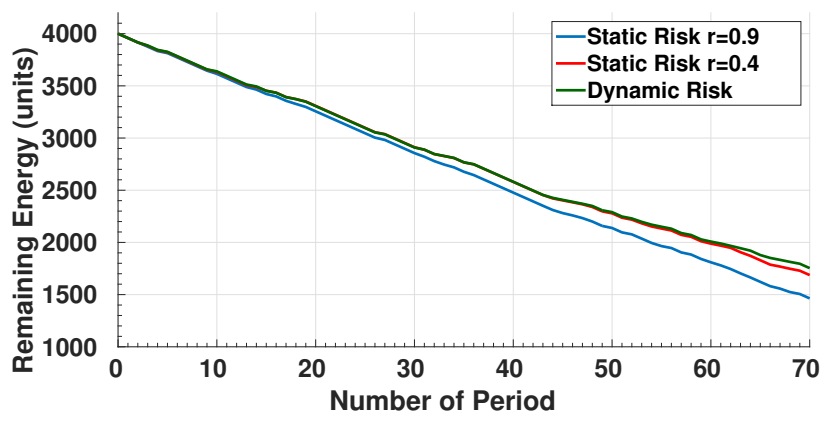

(a) Patient 1: Energy Consumption of the HR sensor node over 70 periods

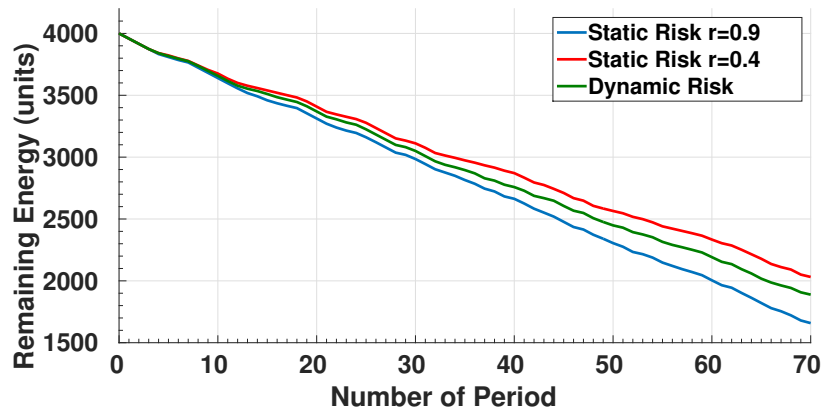

(b) Patient 2: Energy Consumption of the HR sensor node over 70 periods

Fig. 6: Comparison of the HR sensor node's remaining energy at the end of 70 periods between 3 different cases.

performed at the level of each sensor node for the three scenarios. Theoretically, if the Fisher Test null hypothesis is rejected for all 70 periods then a data reduction of only $30 \%$ is acheived given that the maximum sampling rate is $S R_{\max }=1$ sample/1.42 sec (corresponding to 70 samples out of 100 per period). Whereas, if the Fisher Test null hypothesis is accepted for all 70 periods with $F=0$ then a maximum data reduction of $70 \%$ is acheived given that the minimum sampling rate is $S R_{\max }=1 \mathrm{sample} / 3.33 \mathrm{sec}$ (corresponding to 30 samples out of 100 per period) (cf. Algorithm 1). The results show that for both patients and for all the monitored vital signs the percentage of data reduction is bounded by $30 \%$ and $70 \%$ such as the data reduction is stricly higher than $30 \%$ demonstrating that the F-test is a suitable statistical test for this type of medical data. As shown in Table I, a total data reduction of about $48 \%$ compared to the original dataset has been acheived in our approach, of $43 \%$ in the case of a static risk $r=0.9$ and of $47 \%$ in the case of a static risk $r=0.4$. We compare the scores of the measurements of the sampled datasets to the ones of the original dataset in each of the three scenarios, while taking the time granularity of the original dataset $(1 \mathrm{sec})$. We choose the mean-squared error to measure the average of the squares of the errors or deviations given that $M S E=\frac{1}{M \times N} \sum_{i=1}^{M} \sum_{j=1}^{N}(a(i, j)-b(i, j))^{2}$ where $\mathrm{M}$ is the total of monitored vital signs, $\mathrm{N}$ is the total of measurements for each vital sign during the simulation, $a$ is the score of the $j^{\text {th }}$ measurement of the $i^{\text {th }}$ vital sign in the original
TABLE I: Data reduction performed for each monitored vital sign of record s01840-3454-10-24-18-46nm from MIMIC II compared to original dataset in 3 different cases.

\begin{tabular}{|c|c|c|c|c|c|}
\hline Risk Level Setup & HR & SpO2 & BLOODT & Resp & ABPSys \\
\hline Static Risk $r=0.9$ & $40 \%$ & $44 \%$ & $65 \%$ & $34 \%$ & $34 \%$ \\
Static Risk $r=0.4$ & $45 \%$ & $48 \%$ & $65 \%$ & $37 \%$ & $37 \%$ \\
Dynamic Risk & $47 \%$ & $48 \%$ & $65 \%$ & $38 \%$ & $38 \%$ \\
\hline
\end{tabular}

TABLE II: Mean squared error between original dataset and sampled dataset of patient s01840-3454-10-24-18-46nm from MIMIC II for 3 different scenarios.

\begin{tabular}{|c|c|}
\hline Risk Level Setup & MSE \\
\hline Static Risk $r=0.9$ & 0.0128 \\
Static Risk $r=0.4$ & 0.0144 \\
Dynamic Risk & 0.0145 \\
\hline
\end{tabular}

TABLE III: Percentage of detected critical changes compared to original dataset of patient s01840-3454-10-24-18-46nm from MIMIC II for 3 different scenarios.

\begin{tabular}{|c|c|c|c|c|c|}
\hline Risk Level Setup & HR & SpO2 & BLOODT & Resp & ABPSys \\
\hline \hline $\begin{array}{c}\text { Static Risk } \\
r=0.9\end{array}$ & $76 \%$ & $97 \%$ & $80 \%$ & $97 \%$ & $86 \%$ \\
$\begin{array}{c}\text { Static Risk } \\
r=0.4\end{array}$ & $72 \%$ & $96 \%$ & $80 \%$ & $97 \%$ & $85 \%$ \\
Dynamic Risk & $69 \%$ & $95 \%$ & $80 \%$ & $97 \%$ & $86 \%$ \\
\hline
\end{tabular}

dataset and $b$ is the score of the $j^{\text {th }}$ measurement of the $i^{\text {th }}$ vital sign in the sampled dataset. The results (cf. Table II) show that the MSE of the three scenarios are very close to 1 , thus the loss of information is negligible. Our approach has reduced the amount of sampled data 5\% more than the case of static risk $r=0.9$ with a MSE difference of 0.0017 . Table III shows the percentage of critical events detected over 70 periods compared to the critical events recorded in the original dataset. As we can see, the percentage is greater than about $70 \%$ for all vital signs for the three scenarios. However, it is important to note that the percentage of critical events in the original dataset for each of the HR, SpO2, BlOODT, RESP and ABPSys which do not last more than 2 seconds are respectively $38 \%, 1 \%, 20 \%, 5 \%$ and $17 \%$. Thus, in three scenarios and for all vital signs, all of the persistant critical events have been detected. Finally, Figure 7 shows the HR sensor node's totals for each score for the 3 different scenarios in comparison with the totals of the original dataset. As we can see, although our proposed approach has reduced the amount of HR sampled data to $47 \%$ (cf. Table I), the totals of all scores were conserved. Consequently, our proposed approach allows a sensor node to dynamically adapt its risk level over time based on the state of the vital sign it is monitoring as well as the overall health condition of the patient. Fixing the risk level to a high value when it is not needed increases the energy consumption and the amount of sampled data over time whereas in some scenarios, fixing the risk level to a low value can increase the loss of information and some critical events can be passed unseen. 


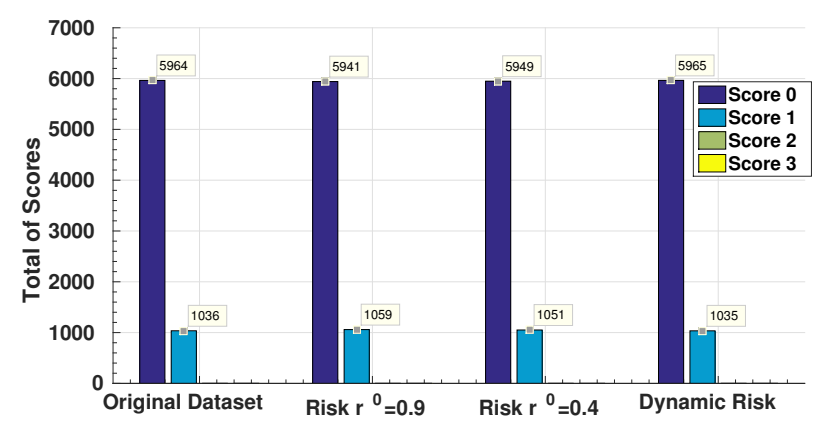

Fig. 7: The HR sensor node's totals for each score for three different scenarios in comparison with the original dataset.

\section{CONClusion}

In this paper, an adaptive sampling rate approach using dynamically adapted risk level has been proposed. Since the patient's health condition, remotly and continously monitored using a WBSN, changes over time, we have proposed to adapt the risk level of each sensor node based on the overall health condition of the patient and the state of their corresponding vital sign. The risk level represents the degree of monitoring importance given to a vital sign regarding the severity level of the patient's health condition. The results show that our approach, compared to an adaptive sampling rate approach with static risk levels, adapts the amount of sampled data and energy consumption according to the patient's status while preserving information about critical events as well as about the changing condition of the vital sign whether it is deteriorating or improving. As a future work, we intend to study other statistical tests which can be applied on medical data to study the variations of the measurements, propose other types of behavior function and compare them with our proposed adaptive sampling rate scheme. Moreover, a real implementation of the proposed approach is intended to be achieved in order to validate its performance on real-case scenarios.

\section{ACKNOWLEDGMENT}

This project has been performed in cooperation with the Labex ACTION program (contract ANR-11-LABX-0001-01). This project has been funded with support from the National Council for Scientific Research in Lebanon.

\section{REFERENCES}

[1] Carmen CY Poon, Benny PL Lo, Mehmet Rasit Yuce, Akram Alomainy, and Yang Hao. Body sensor networks: In the era of big data and beyond. Biomedical Engineering, IEEE Reviews in, 8:4-16, 2015.

[2] Ivan Miguel Pires, Nuno M Garcia, Nuno Pombo, and Francisco FlórezRevuelta. From data acquisition to data fusion: a comprehensive review and a roadmap for the identification of activities of daily living using mobile devices. Sensors, 16(2):184, 2016.

[3] Giancarlo Fortino, Stefano Galzarano, Raffaele Gravina, and Wenfeng Li. A framework for collaborative computing and multi-sensor data fusion in body sensor networks. Information Fusion, 22:50-70, 2015.

[4] Shahina Begum, Shaibal Barua, and Mobyen Uddin Ahmed. Physiological sensor signals classification for healthcare using sensor data fusion and case-based reasoning. Sensors, 14(7):11770-11785, 2014.
[5] Min Chen, Sergio Gonzalez, Athanasios Vasilakos, Huasong Cao, and Victor CM Leung. Body area networks: A survey. Mobile networks and applications, 16(2):171-193, 2011.

[6] Tifenn Rault, Abdelmadjid Bouabdallah, and Yacine Challal. Energy efficiency in wireless sensor networks: A top-down survey. Computer Networks, 67:104-122, 2014.

[7] Christian Salim, Abdallah Makhoul, Rony Darazi, and Raphaël Couturier. Adaptive sampling algorithms with local emergency detection for energy saving in wireless body sensor networks. In Network Operations and Management Symposium (NOMS), 2016 IEEE/IFIP, pages 745-749. IEEE, 2016.

[8] Michele Magno, Tommaso Polonelli, Filippo Casamassima, Andres Gomez, Elisabetta Farella, and Luca Benini. Energy-efficient context aware power management with asynchronous protocol for body sensor network. Mobile Networks and Applications, pages 1-11, 2016.

[9] Shih-Yeh Chen, Chin-Feng Lai, Ren-Hung Hwang, Ying-Hsun Lai, and Ming-Shi Wang. An adaptive sensor data segments selection method for wearable health care services. Journal of medical systems, 39(12):194, 2015.

[10] Hui Wang, Hyeok-soo Choi, Nazim Agoulmine, M Jamal Deen, and James Won-Ki Hong. Information-based energy efficient sensor selection in wireless body area networks. In Communications (ICC), 2011 IEEE International Conference on, pages 1-6. IEEE, 2011.

[11] Hossein Mamaghanian, Nadia Khaled, David Atienza, and Pierre Vandergheynst. Compressed sensing for real-time energy-efficient ecg compression on wireless body sensor nodes. IEEE Transactions on Biomedical Engineering, 58(9):2456-2466, 2011.

[12] Oliver Faust, U Rajendra Acharya, Jianguo Ma, Lim Choo Min, and Toshiyo Tamura. Compressed sampling for heart rate monitoring. Computer methods and programs in biomedicine, 108(3):1191-1198, 2012.

[13] Shancang Li, Li Da Xu, and Xinheng Wang. A continuous biomedical signal acquisition system based on compressed sensing in body sensor networks. IEEE transactions on industrial informatics, 9(3):1764-1771, 2013.

[14] Aosen Wang, Feng Lin, Zhanpeng Jin, and Wenyao Xu. A configurable energy-efficient compressed sensing architecture with its application on body sensor networks. IEEE Transactions on Industrial Informatics, 12(1):15-27, 2016.

[15] Yu Jiang, Pengliu Tan, Houbing Song, Binhua Wan, Mohammad Hosseini, and Lui Sha. A self-adaptively evolutionary screening approach for sepsis patient. In Computer-Based Medical Systems (CBMS), 2016 IEEE 29th International Symposium on, pages 60-65. IEEE, 2016.

[16] Carol Habib, Abdallah Makhoul, Rony Darazi, and Christian Salim. Self-adaptive data collection and fusion for health monitoring based on body sensor networks. IEEE Transactions on Industrial Informatics, 12(6):2342-2352, 2016

[17] K. Baskaran Ragesh .G.K. A survey on futuristic health care system: Wban. Procedia Engineering, 30(0):889 - 896, 2012.

[18] National early warning score. http://www.rcplondon.ac.uk/resources/ national-early-warning-score-news, 2012. Accessed: 2016-5-10.

[19] Abdallah Makhoul, David Laiymani, Hassan Harb, and Jacques M Bahi. An adaptive scheme for data collection and aggregation in periodic sensor networks. International journal of sensor networks, 18(1-2):6274, 2015.

[20] Carol Habib, Abdallah Makhoul, Rony Darazi, and Raphaël Couturier. Multisensor data fusion for patient risk level determination and decisionsupport in wireless body sensor networks. In Proceedings of the 19th ACM International Conference on Modeling, Analysis and Simulation of Wireless and Mobile Systems, pages 221-224. ACM, 2016.

[21] Physionet. https://www.physionet.org/, 2000 - present. Accessed: 201610-4.

[22] Carol Habib, Abdallah Makhoul, Rony Darazi, and Raphaël Couturier. Health risk assessment and decision-making for patient monitoring and decision-support using wireless body sensor networks. Information Fusion, Submitted. March 2017.

[23] Michele Magno, Tommaso Polonelli, Filippo Casamassima, Andres Gomez, Elisabetta Farella, and Luca Benini. Energy-efficient context aware power management with asynchronous protocol for body sensor network. Mobile Networks and Applications, pages 1-11, 2016. 